\title{
The gluon structure of hadrons and nuclei from lattice QCD
}

\author{
Phiala Shanahan ${ }^{1,2,3, \star}$ \\ ${ }^{1}$ Center for Theoretical Physics, Massachusetts Institute of Technology Cambridge, MA 02139, USA \\ ${ }^{2}$ Department of Physics, College of William and Mary, Williamsburg, VA 23187-8795, USA \\ ${ }^{3}$ Thomas Jefferson National Accelerator Facility, 12000 Jefferson Avenue, Newport News, VA 23606, USA
}

\begin{abstract}
I discuss recent lattice QCD studies of the gluon structure of hadrons and light nuclei. After very briefly highlighting new determinations of the gluon contributions to the nucleon's momentum and spin, presented by several collaborations over the last year, I describe first calculations of gluon generalised form factors. The generalised transversity gluon distributions are of particular interest since they are purely gluonic; they do not mix with quark distributions at leading twist. In light nuclei they moreover provide a clean signature of non-nucleonic gluon degrees of freedom, and I present the first evidence for such effects, based on lattice QCD calculations. The planned ElectronIon Collider, designed to access gluon structure quantities, will have the capability to test this prediction, and measure a range of gluon observables including generalised gluon distributions and transverse momentum dependent gluon distributions, within the next decade.
\end{abstract}

\section{Introduction}

A detailed picture of the quark structure of nucleons has emerged over the last half-century, through increasingly precise measurements of quantities such as the nucleon electromagnetic form factors and quark momentum distribution functions, as well as many spin-dependent aspects of nucleon structure $[1,2]$. In concert, lattice QCD calculations of such quantities have become sophisticated and precise, often with fully-controlled uncertainties. In contrast, the gluonic structure of nucleons (beyond the gluon density) remains relatively unexplored. Gluon structure quantities are much harder to measure experimentally than their quark analogues, as the gluon does not couple to electromagnetic probes and thus other approaches must be taken, for example using the Drell-Yan process. This lack of information has inspired the proposal and planning of an Electron-Ion Collider (EIC), which was the highest priority for new construction in the 2015 NSAC long range plan of the US nuclear physics community [8]. The primary mission of this new experiment $[9,10]$ is to determine aspects of the gluon structure of hadrons and nuclei with unprecedented precision. Complementary experiments at Jefferson National Accelerator Facility [11] and at the LHC [12] also aim to study various gluon distributions. In this light, lattice QCD calculations of gluon structure quantities have taken on new importance, and there has been significant progress on this front over the last year [5, 13-16], expanding and building on pioneering lattice QCD studies of the unpolarised gluonic structure of the pion

${ }^{\star}$ Speaker, e-mail: peshanahan@wm.edu 
and nucleon [17-19]. On a decade-long timescale, such calculations will guide the design requirements of the EIC by revealing the magnitude of observables that are key targets of the experimental program. These include the gluon generalised parton distributions (GPDs) and transverse-momentum distributions (TMDs) of the nucleon, as well as nuclear physics quantities such as gluon analogues of the EMC effect and 'exotic glue' observables which encode non-nucleonic gluon structure in nuclei. Furthermore, there is the exciting possibility for fully-controlled lattice QCD calculations of these gluon observables to serve as QCD predictions ahead of first data-taking at the EIC.

Here I discuss some of the recent progress in lattice QCD calculations of several aspects of the gluon structure of hadrons and nuclei, addressing the questions:

- How much do gluons contribute to static properties of the nucleon, e.g., momentum, mass and spin?

- What is the three-dimensional distribution of gluons in the nucleon, i.e., TMDs, GPDs? How does the 'gluon radius' of a nucleon compare with an equivalent 'quark radius' or the charge radius?

- How is the gluon structure of a nucleon modified in a nucleus i.e., what are the magnitudes of gluon analogues of the EMC effect?

\section{Gluon structure of the hadrons}

Several collaborations have performed novel calculations of gluon structure observables in hadrons over the last year. In particular, the gluon contributions to the nucleon's momentum $[5,13]$ and spin $[5,6]$ have been directly calculated at quark masses corresponding to the the physical value of the pion mass, and the first lattice QCD calculations of moments of gluon GPDs $[14,15]$ have been performed. These calculations are summarised in the following sections.

\subsection{Nucleon momentum fraction}

Since the 2016 lattice conference, there have been two direct calculations of the gluon light cone momentum fraction $\langle x\rangle_{g}$ in the nucleon at quark masses corresponding to the physical value of the pion mass $[5,13]$. Previously, a number of calculations of this quantity had been performed at largerthan-physical values of the quark masses, paving the way for these new results [18, 20, 21]. Notably, Ref. [5] includes a complete decomposition of the nucleon's momentum into quark and gluon connected and disconnected contributions, with the momentum sum rule not imposed, but found to be satisfied within uncertainties as shown in Fig. 1. The gluon contribution in the $\overline{\mathrm{MS}}$ scheme at $2 \mathrm{GeV}$ was found to be $27(3) \%$. This result is in slight tension with that from the phenomenological CT14NNLO parton distribution fits [22]. This is also shown in Fig. 1, along with previous lattice QCD results and the preliminary result from Ref. [13] which also includes calculations at quark masses corresponding to the physical value of the pion mass. It will be interesting to see whether this tension persists as calculations are further improved.

\subsection{Nucleon spin}

Since the original discovery of the so-called proton spin crisis - the realisation that quarks carry only a relatively small fraction of the proton spin - by the European Muon Collaboration (EMC) [23], there has been enormous effort to resolve this puzzle. Over the last year, important progress has been made on this front from lattice QCD, with complete and fully-controlled determinations of various nucleon spin contributions, including the poorly-known gluon contributions $[5,6]$. 


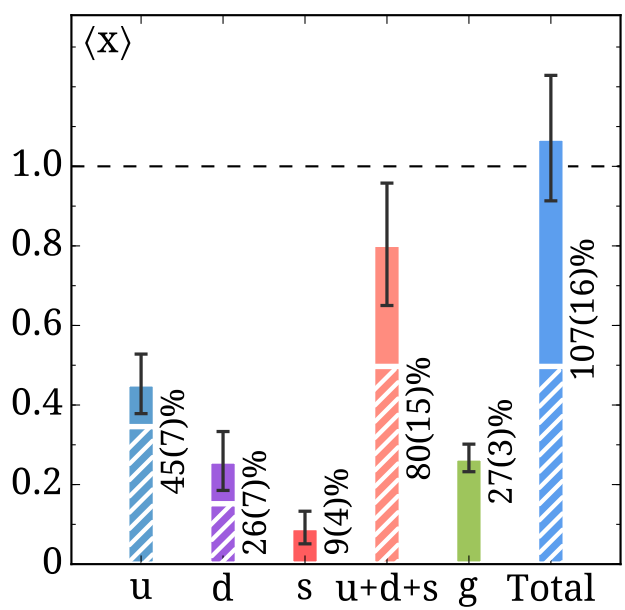

(a) Figure taken from Ref. [5], which includes a calculation of the gluon contribution to the nucleon momentum (green vertical bar labelled ' $\mathrm{g}$ ') at the quark masses corresponding to the physical value of the pion mass.

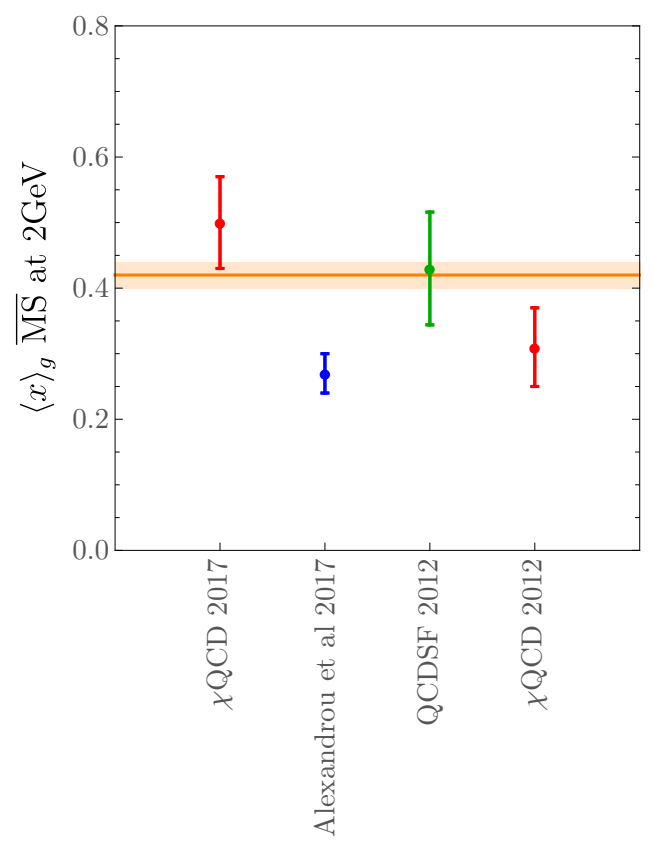

(b) Summary figure, showing results from Refs. [5, 13, 18, 21]. The horizontal band shows the phenomenological result from the CT14NNLO parton distribution global fits from Ref. [22].

Figure 1. Gluon contribution to the nucleon momentum fraction, from recent lattice QCD calculations.

There is a continuum of ways to decompose the nucleon spin into contributions from quark and gluon helicity and spin or orbital angular momentum. Two commonly-used decompositions are the Ji decomposition:

$$
J_{N}^{\mathrm{Ji}}=\sum_{q=u, d, s, c \ldots}\left(\frac{1}{2} \Delta \Sigma_{q}+L_{q}\right)+J_{g},
$$

where $\Sigma_{q}$ denotes the quark helicity, $L_{q}$ refers to the quark angular momentum, and $J_{g}$ represents the gluon spin, and the Jaffe-Manohar decomposition:

$$
J_{N}^{\mathrm{JM}}=\sum_{q=u, d, s, c \ldots}\left(\frac{1}{2} \Delta \Sigma_{q}+\mathcal{L}_{q}\right)+\Delta g+\mathcal{L}_{g},
$$

where $\Delta g$ is known as the gluon helicity, respectively. The last year has seen not only controlled lattice QCD calculations of the gluon contributions in each decomposition [5, 6], but notable new discussion of the difference and interpolation between these different decompositions of the nucleon spin from a lattice QCD perspective [24].

All terms in the Ji spin decomposition are gauge invariant, and the total quark contribution to the nucleon spin in this decomposition is measurable through deeply virtual compton scattering. There is no further decomposition of the gluon spin contribution into spin or orbital angular momentum components, but the total gluon spin can be calculated in lattice QCD using standard techniques. This 


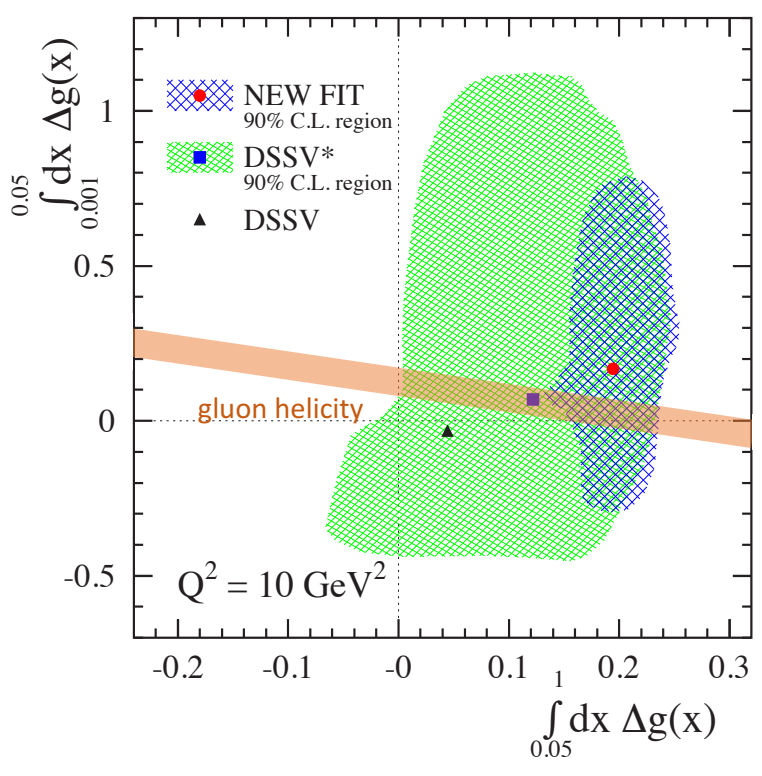

Figure 2. Figure modified from Ref. [26], showing the results of a global QCD analysis of polarised parton distributions, to include the lattice QCD result of Ref. [6] as the orange band.

year, such a calculation was performed at quark masses corresponding to the physical pion mass [5], in a study that presented a complete decomposition of the nucleon spin in this basis. It is notable that the percentage gluon contribution to the nucleon spin found in this study-about $27 \%$-is the same percentage gluon contribution to the momentum fraction of the nucleon found in the same set of calculations, as discussed in the previous section. Importantly, the total spin of the nucleon in this study, with all contributions determined separately, was consistent with $\frac{1}{2}$, as required by the spin sum rule.

The Jaffe-Manohar approach gives a canonical partonic, but gauge-variant, decomposition of the nucleon spin into quark and gluon spin and orbital angular momentum contributions in the infinite momentum frame in the light-cone gauge. The spin contributions in this decomposition are measurable through polarised deep inelastic scattering. As it is defined in the light cone frame, the Jaffe-Manohar gluon helicity is not directly accessible to lattice QCD defined on a Euclidean spacetime. In Ref. [6], the large momentum effective theory [25] approach was used to access the gluon helicity from lattice QCD for the first time. In this approach, matrix elements that define the gluon helicity in the infinite momentum limit are calculated, and the extrapolation to large momentum is performed. In the calculation of Ref. [6], which included several lattice spacings and volumes including an ensemble with quark masses corresponding to the physical pion mass, the most significant uncertainty arose from the large-momentum extrapolation. The result is compared with phenomenological results from a global analysis of polarised parton distributions [26] in Fig. 2. The determinations are entirely consistent, with the lattice QCD result providing a significantly improved constraint on the total gluon helicity. 


\subsection{D gluon structure}

To completely describe the gluon distribution in the nucleon-to achieve real three-dimensional tomography of the nucleon as a composite object-involves knowledge of both the motion of gluons inside the nucleon and of their spatial distribution. This information, for both quarks and gluons, is encoded in a number of distribution functions. The generalized parton distributions (GPDs) describe the distribution of quarks and gluons both in the transverse plane and in the longitudinal direction [27], encompassing and generalizing the information carried by the parton distribution functions (PDFs) and the elastic electromagnetic form factors. The GPDs are functions of both the squared momentum transfer $t$ at which the nucleon is probed, and the longitudinal momentum fraction $x$. Reducing the dimensionality of these distributions by integrating over $x$ defines the Mellin moments of the GPDs (specifically, the tower of $x^{n}$-weighted integrals over the longitudinal momentum fraction $x$ of the GPDs defines a tower of moments that together contain all of the information of the GPDs). These moments are parametrised as generalised form factors (GFFs), observables that can both be studied from lattice QCD using standard lattice calculations of hadronic matrix elements of local operators $[28,29]^{1}$ and indirectly constrained by experimental measurements at, for example, the planned EIC.

Measurements and calculations of the spin-independent gluon distributions in particular can reveal the answer to a tantalizing question: "What is the gluon radius of a nucleon, and how does it compare to the quark radius?" The charge radius of the proton is a fundamental quantity describing nucleon structure, and resolving the $7 \sigma$ discrepancy between determinations of the radius from electron scattering experiments and electronic hydrogen spectroscopy on the one hand and measurements of muonic hydrogen $[40,41]$ on the other-termed the 'proton radius puzzle' - is a high priority for the hadronic physics community [42-46]. In principle, the gluonic radius of the proton is no less important or interesting, and need not be the same, but it is completely unknown from experiment. Models and phenomenology offer little intuition on this topic ${ }^{2}$. For example, guided by a bag model of the nucleon, where gluons expand to fill the bag, one might expect the gluon radius to be larger than the quark radius. On the other hand, drawing on the constituent quark model where gluons hide inside dressed constituent quarks, one could expect the quark and gluon radii to be similar. A different picture again is painted by lattice QCD calculations with infinitely heavy quarks which reveal gluon flux tubes binding quarks together [47], suggesting that the gluon radius is smaller than the quark radius. Lattice QCD calculations of GFFs can offer some insight into this fundamental question.

Recently, the momentum-dependence of some of the GFFs of the $\phi(\bar{s} s)$ meson was calculated using lattice methods $[14,15]$, providing the first information on the multidimensional gluon structure inside any hadron. These studies were performed with heavy values of the light quark masses corresponding to a pion mass of $450 \mathrm{MeV}$, but a value of the strange quark mass yielding a closeto-physical $\phi$ mass. Through direct calculations of matrix elements of local gluonic operators, the momentum-dependence of the lowest terms in the towers of the spin-independent and transversity GFFs was investigated. The latter GFFs, encoding how much more momentum of a transversely polarised particle is carried by gluons aligned rather than perpendicular to it in the transverse plane, are particularly clean since they have no mixing with quark distributions until subleading twist and

\footnotetext{
${ }^{1}$ Until very recently it was not known how one might calculate $x$-dependent quantities such as PDFs and GPDs directly using lattice QCD [30]; deep inelastic scattering and related processes are dominated by physics that is light-like, and as such are inaccessible in Euclidean space. Over the last few years, however, several methods have been suggested to access the $x$-dependence of PDFs using lattice QCD [30-32]. These approaches necessarily involve a rotation to a 'quasi' or 'pseudo' PDF, defined in Euclidean space, and an extrapolation back to the physical quantity in the large-momentum limit. While these calculations are in early stages, they are promising $[33,34]$. Similarly, the first lattice QCD calculations related to TMDs have been performed in the last few years [35-39].

${ }^{2}$ This question was first brought to my attention in discussions with A. Deshpande.
} 


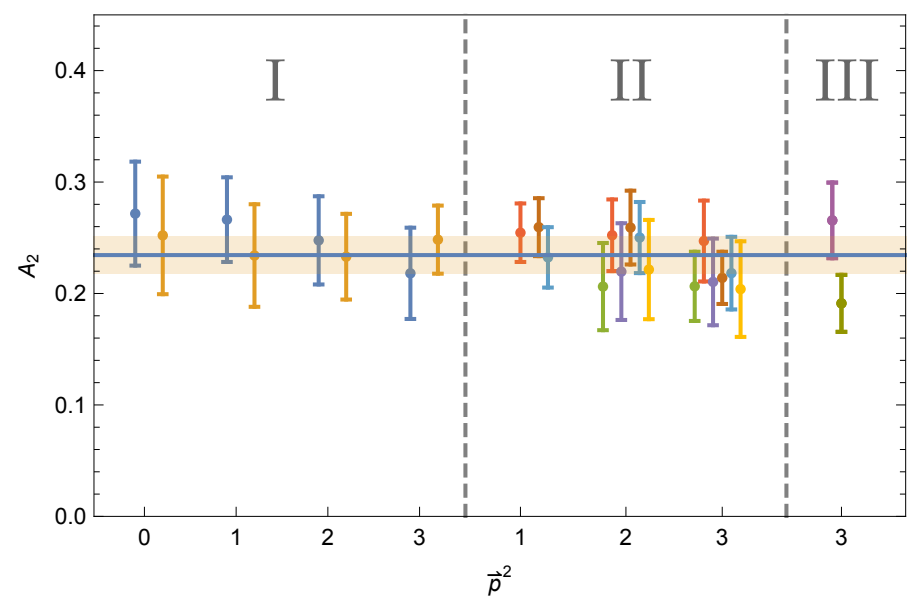

Figure 3. Figure taken from Ref. [14]. Reduced matrix element encoding the lowest moment of the forwardlimit gluon transversity distribution (unrenormalised), extracted from calculations at different boost momenta $\vec{p}^{2}$. Results in sections I, II and III of the figure are determined from vectors in different irreducible representations of the hypercubic group (and can differ by discretisation artefacts). Different colours, offset on the horizontal access for clarity, denote different vectors in each irreducible representation. The horizontal band is shown to guide the eye.

hence provide a direct probe of gluonic structure [48]. In the nucleon (or in hadrons or nuclei with spin $<1$ ), the transversity GFFs decouple from the gluon matrix elements in the forward limit as a result of angular momentum conservation. For this reason, the $\phi$ meson, as the simplest spin- 1 system (therefore having an accessible forward-limit transversity distribution), was chosen for this first investigation. Experimentally, the gluon transversity distributions can be probed through distinct angular dependence of the cross-section in deeply-virtual Compton scattering (DVCS) [48].

In the forward limit, the magnitude of the gluon transversity distribution can be related to the magnitudes of the spin-independent and spin-dependent gluon distributions by a Soffer-type bound [49]. These bounds, which are positivity constraints arising from the positivity of the scattering matrix, were first studied in moment space on the lattice in Ref. $[50,51]$ for the quark distributions of spin$1 / 2$ particles. It is found in Ref. [14] that the Soffer bound for the first moment of the gluon distributions [52-55] in the spin-1 $\phi$ meson is saturated to $80 \%-100 \%$ (subject to some caveats regarding renormalisation of the GFFs), similar to the lattice QCD results found for the first two moments of the quark distributions of the nucleon [50, 51]. Reference [14] also included a detailed study of different irreducible representations of the hypercubic group (chosen such that the breaking of rotational symmetry does not induce mixing with lower or same-dimensional operators), illustrated in Fig. 3, which suggested negligible discretisation artefacts. It was also found in that study that a smearing prescription, for example Wilson flow [56] or HYP smearing [57] applied to the links in the gluon operator, was necessary to reduce noise in the calculation. While the results in Ref. [14] are unrenormalised, a perturbative renormalisation has recently been computed for similar gluonic operators $[18,58]$.

Away from the forward limit, a large number of gluon GFFs contribute to the matrix elements of local gluonic operators in the $\phi$ meson; there are 7 spin-independent gluon GFFs for the lowest moment in the infinite tower that describes the full GPDs (with more contributing at each successive rung in the tower), and there are 8 for the transversity case. Because of this large number of GFFs, 


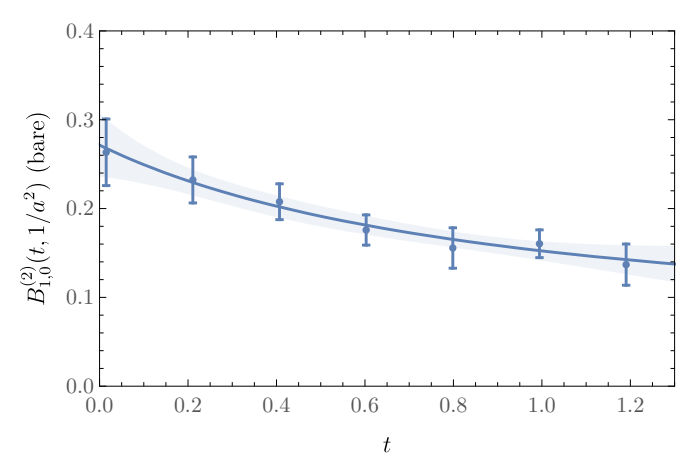

(a) Gluon transversity generalised form factor.

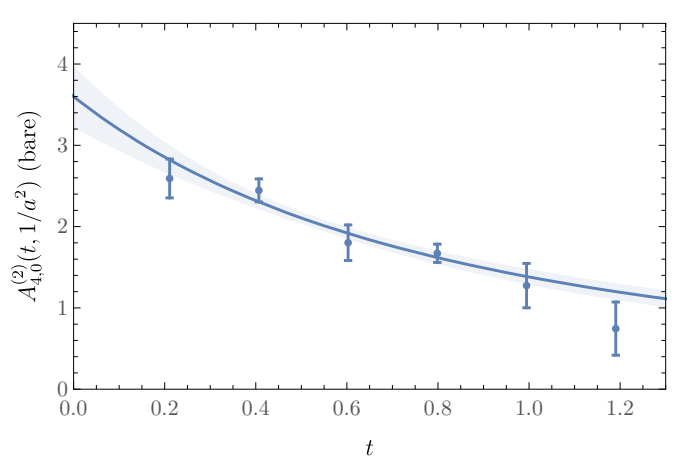

(b) Spin-indepenent gluon generalised form factor.

Figure 4. Examples of generalised gluon form factors of the $\phi$ meson; figures taken from Ref. [15]. The horizontal axes show the squared momentum transfer $t$ in $\mathrm{GeV}^{2}$, while the bands show fits to generalised dipole forms.

not all could be determined from the lattice QCD calculations of Ref. [15]. In particular, at some (discretised) values of the momentum transfer, some of the GFFs contribute to the calculated matrix elements with coefficients suppressed by many orders of magnitude, and could not be extracted given the statistical precision of the calculations. A complete extraction will require more precise data at many different sets of initial and final momenta giving the same momentum transfer. Despite the challenges of the calculation, the momentum-dependence ( $t$-dependence) of one of the eight gluon transversity GFFs, and three of the seven unpolarised gluon GFFs of the $\phi$ meson, was determined. Examples of these are shown in Fig. 4. The dipole-like momentum-dependence of the form factors, revealing qualitatively similar shapes as seen for the electromagnetic form factors which describe the spatial quark distributions and charge radius, provides the first information about the extended gluonic structure of the $\phi$. All of the computational and analysis techniques developed in that study will carry over to future calculations of gluon GFFs in the phenomenologically relevant case of the nucleon.

Based on the calculations of gluon GFFs in Ref. [15], we can return to the question of a gluonic radius, and how such a radius compares with the corresponding quark radius. The clean dipolelike momentum-dependence of all of the form factors which could be obtained (including those not reproduced here in Fig. 4) suggests that the familiar parameterisation of each radius in terms of a dipole mass is sensible here. Moreover, the GFFs of the spin-independent quark distribution have an identical decomposition to the gluon GFFs and so quark and gluon radii can be compared one-toone in a natural way. Making this comparison (see Fig. 5), it becomes clear that each of the three gluon radii that were determined in Ref. [15] is different from the corresponding quark radius: one larger, one smaller, and one approximately the same. Further interpretation will require a change of the basis of GFFs used in Ref. [15] to a basis with more natural interpretations (e.g., a multipole decomposition).

\section{Gluon structure of nuclei}

In the intensity-frontier era, where experiments such as long baseline neutrino programs and dark matter direct detection experiments typically use nuclear targets, understanding the structure of nuclei from QCD is of increasing importance. While it has been known for decades that the PDFs of large 


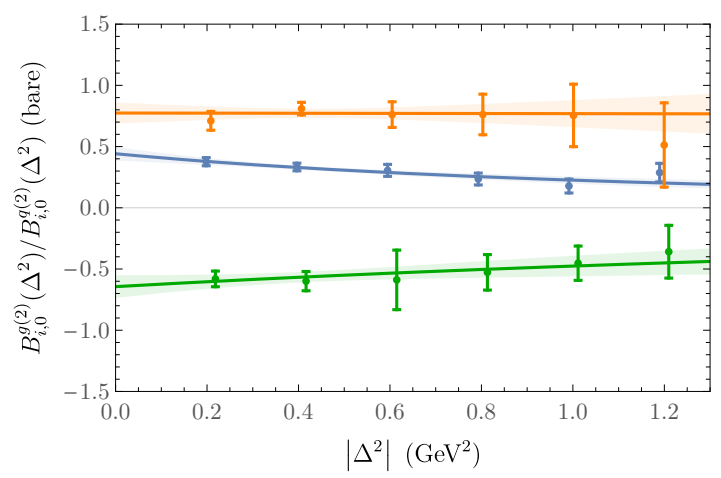

Figure 5. Figure taken from Ref. [15]. Ratios of a subset of the gluon spin-independent GFFs of the $\phi$ meson to the corresponding quark spin-independent GFFs (from top to bottom, the bands correspond to the GFFs $i=$ $\{1,2,4\})$. The horizontal axes show the squared momentum transfer $\left|\Delta^{2}\right|=t$ in $\mathrm{GeV}^{2}$, while the bands show fits to generalised dipole forms. The green band (lowest on the figure) has been re-scaled by a factor of $\frac{1}{10}$ for clarity.

nuclei are not simply determined by a sum of the PDFs of the constituent nucleons [23], known as the EMC effect, the QCD origins of the effect are not yet fully understood [59-65]. An analogue of the EMC effect for gluons, in which the distributions of gluons in a nucleus differ from the sum of the gluon distributions in the constituent protons and neutrons [66], should in principle exist but has not been observed and would be a milestone discovery of the EIC [67]. A prediction of the magnitude of this 'gluonic EMC effect' from lattice QCD would be influential in the planning of the EIC program.

Recently, the first lattice QCD calculation of aspects of the gluon structure of light nuclei was performed [16]. That study presented results for the gluon momentum fraction of the nucleon, spin- 0 dinucleon, spin-1 deuteron, and spin- $\frac{1}{2}$ triton, using two ensembles with light quark masses chosen such that $m_{\pi} \sim 450 \mathrm{MeV}$ and $m_{\pi} \sim 806 \mathrm{MeV}^{3}$. Given that the magnitude of the EMC effect in the quark momentum fraction of light nuclei from phenomenological parameterisations of the quark and gluon distributions $[22,72,73]$ is typically at the level of $5 \%$, the momentum sum rule dictates that the EMC effect in the gluon momentum fraction must be of the same magnitude and opposite sign. Of course, at the larger-than-physical values of the quark masses used in the simulations of Ref. [16], this effect could be larger or smaller than in nature. With the statistics of that work, no EMC effect could be resolved (see Fig. 6). Resolving an EMC effect of the same magnitude as it is expected to be in nature would require few-percent determinations of the proton and nuclear gluon momentum fractions, corresponding to an order of magnitude more statistics. Higher moments of the gluon PDFs could display larger (or smaller) EMC-type effects, not being constrained by momentum conservation. These are more difficult to access from lattice QCD since they are related to matrix elements of local gluonic operators with additional derivatives, but present promising avenues for further study.

The facility of lattice QCD, and the future EIC, to study gluon distributions of nuclei is not limited to the momentum fraction. Measurements of exotic glue observables displaying non-nucleonic features of a nucleus are a particularly tantalising prospect. These effects, illustrated in Fig. 7, describe contributions to nuclear structure from gluons that can not be associated with individual nucleons in a nucleus. That is, while for many purposes nuclei can be described as a collection of modified nucle-

\footnotetext{
${ }^{3}$ The spectrum and many properties of light nuclei on these ensembles have been studied in detail previously [68-71].
} 


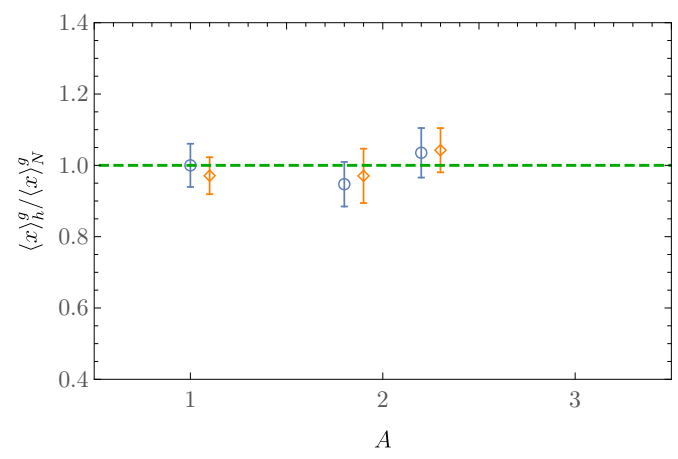

(a) Results at $m_{\pi} \sim 450 \mathrm{MeV}$.

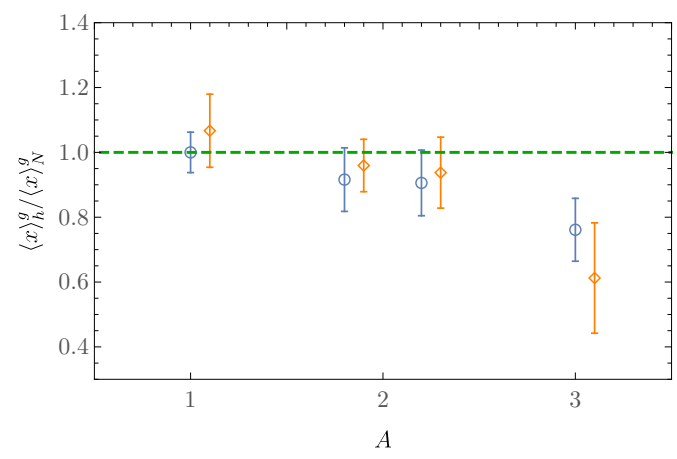

(b) Results at $m_{\pi} \sim 806 \mathrm{MeV}$.

Figure 6. Figures taken from Ref. [16], showing the ratios of the gluon momentum fraction in light nuclei to the central value of that in the nucleon. The $A=1$ results are for the nucleon, the spin- 1 deuteron and spin- 0 dinucleon are shown at $A=2$, and the triton is shown at $A=3$ on the horizontal axis. The blue circles and orange diamonds denote results obtained using different lattice operator constructions. The EMC effect would present as deviations of the $A>1$ results from the dashed line shown at 1 .

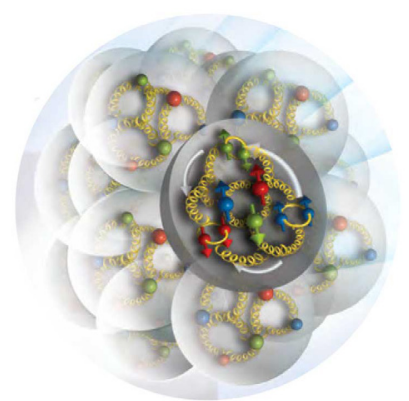

(a) Traditional picture of nuclear structure, with gluon interactions (represented by curly yellow lines) confined to individual nucleons (figure taken from Ref. [9]).

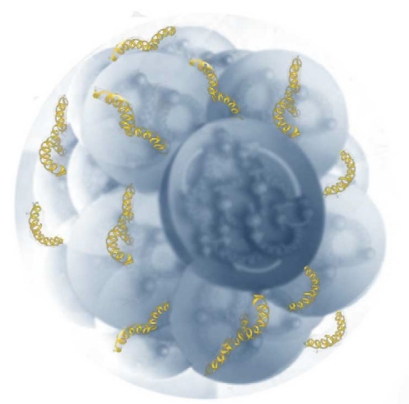

(b) Nucleus with 'exotic' multinucleonic glue effects highlighted.

Figure 7. Artist's interpretation of 'exotic' observables describing multi-nucleonic contributions to the gluon structure of a nucleus.

ons ${ }^{4}$, exotic glue observables directly quantify the breaking of this nucleon-based picture of nuclear structure in nature. There is potential for experiments at Thomas Jefferson National Accelerator Facility [11] to measure exotic glue effects, in particular the gluon transversity structure function in spin $\geq 1$ nuclei, imminently.

\footnotetext{
${ }^{4}$ Indeed, from an EFT perspective such off-shell nucleon effects and two-nucleon interactions with extended currents are related by unitary transformations $[74,75]$.
} 
Reference [16] recently presented the first lattice QCD determination of the gluon transversity structure function in the spin- 1 deuteron state using an ensemble with $m_{\pi}=806 \mathrm{MeV}$. While systematics including discretisation and finite-volume effects, as well as renormalisation and an extrapolation in pion mass, have not yet been addressed, that work provided the first definitive evidence for multi-nucleonic glue effects in a nucleus, albeit at a larger-than-physical value of the pion mass. The extracted result for the gluon transversity matrix element is non-zero to two standard deviations (see Fig. 8), but the significance of the result is far greater; there is no effect other than exotic glue that could cause the appearance of a signal (including the expected exponential contamination from excited states) in the matrix element calculated. The matrix element is safe from mixing with quark distributions at leading twist, and contributions from single nucleon states (even excited single nucleon states) necessarily vanish, unless those states interact and exchange momentum (therefore, a multi-nucleonic effect). For that reason, the result of Ref. [16], although not completely controlled quantitatively, gives a clear qualitative indication of exotic glue. It is also notable that the magnitude of the result is consistent with expectations from large- $N_{c}$ [76-80]; the ratio of the first moment of the gluon transversity distribution to the same moment of the spin-independent gluon distribution in the deuteron is suppressed by an additional factor of $1 / N_{c}^{2}$ as compared with the ratio in a typical compact spin-1 hadron such as the $\phi$ meson [14]. Extensions of this study with fully controlled uncertainties will provide valuable guidance to experimental programs $[9,11]$ seeking to observe this effect in nature for the first time.

\section{Conclusion}

The construction of an electron-ion collider [9] will dramatically improve our ability to probe the gluon structure of hadrons and nuclei, and will complement experimental programs at Thomas Jefferson National Accelerator Facility [11] and at the LHC [12]. In parallel, lattice QCD calculations of gluon structure observables are advancing rapidly, with recent progress making direct calculations of the gluon contributions to observables such as the momentum and spin of the nucleon, at the physical values of the quark masses and with controlled uncertainties, possible for the first time [5, 6, 13]. Complex aspects of the gluon structure of nucleons and nuclei are also being investigated, with the first study of the gluon GFFs encoding moments of gluon GPDs in hadrons performed this year [14, 15], and studies of the $x$-dependence of gluon PDFs and GPDs, as well as gluon TMDs, likely in the near future. Moreover, aspects of the gluon structure of light nuclei have recently been studied from lattice QCD for the first time [16]. Refinements of the calculations of the gluon momentum fraction in light nuclei have the potential to reveal the magnitude of a gluonic analogue of the EMC effect. This effect is a key target of the upcoming EIC program, and its discovery would represent a milestone achievement for that machine. Another particularly exciting recent result is the first definitive indication for 'exotic' multi-nucleonic gluon effects (albeit with uncertainties that are not fully quantified at this stage), revealed through lattice QCD studies of the gluon transversity distribution [16]. It was first pointed out in 1989 [81] that this particular distribution provides a uniquely clean window on gluon effects in nuclei, and it is the target of several planned experimental investigations $[9,11]$.

In summary, lattice QCD calculations of the gluon structure of hadrons and light nuclei are advancing rapidly and complement and extend our understanding of the fundamental structure of nature from experiment. Over the next decade, these results will be influential in the planning and development of experimental programs including the EIC program, and the lattice community has the opportunity to provide controlled QCD predictions for key gluon structure observables ahead of first measurements. 


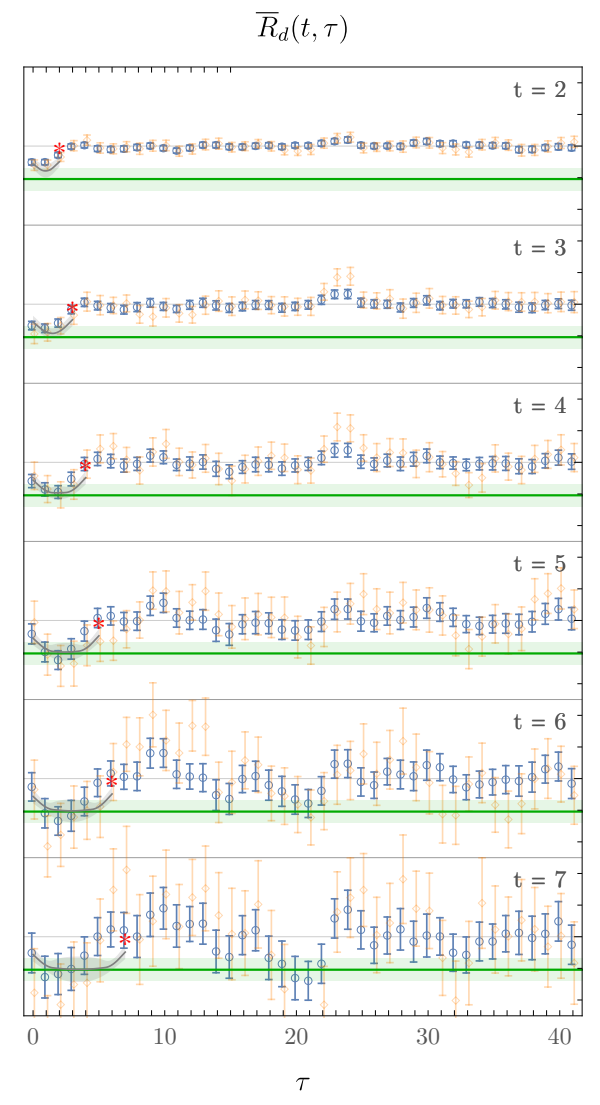

Figure 8. Figure taken from Ref. [16], showing ratios of three-point to two-point functions that reduce to the lowest moment of the gluon transversity distribution in the deuteron for $0 \ll \tau \ll t$, with exponential contamination from excited states at other times. Blue circles and orange diamonds denote results using different source-sink structures, while the red star indicates the sink time $t$. The green bands (repeated on each panel) show the extracted results for the matrix elements from fits in the $t-\tau$ plane using a multi-exponential fit form.

\section{Acknowledgements}

This work is partially supported through contract number DE-AC05-06OR23177 under which JSA operates the Thomas Jefferson National Accelerator Facility. I thank Yi-Bo Yang for sharing preliminary results [13] with me before Lattice 2017 and allowing me to reproduce them here. I also thank Will Detmold, Arjun Gambhir, Dimitra Pefkou, Kostas Orginos, Martin Savage and Michael Wagman for their contributions to the results presented here. 


\section{References}

[1] A. De Roeck, R.S. Thorne, Prog. Part. Nucl. Phys. 66, 727 (2011), 1103.0555

[2] K.A. Olive et al. (Particle Data Group), Chin. Phys. C38, 090001 (2014)

[3] S. Borsanyi et al. (Budapest-Marseille-Wuppertal), Phys. Rev. Lett. 111, 252001 (2013), 1306.2287

[4] E. Berkowitz et al. (2017), 1704.01114

[5] C. Alexandrou, M. Constantinou, K. Hadjiyiannakou, K. Jansen, C. Kallidonis, G. Koutsou, A.V. Avilés-Casco, C. Wiese (2017), 1706.02973

[6] Y.B. Yang, R.S. Sufian, A. Alexandru, T. Draper, M.J. Glatzmaier, K.F. Liu, Y. Zhao, Phys. Rev. Lett. 118, 102001 (2017), 1609.05937

[7] R.S. Sufian, Y.B. Yang, A. Alexandru, T. Draper, J. Liang, K.F. Liu, Phys. Rev. Lett. 118, 042001 (2017), 1606.07075

[8] A. Aprahamian, R. Atcher, H. Caines, G. Cates, J.A. Cizewski, V. Cirigliano, D.J. Dean, A. Deshpande, R. Ent, F. Fahey et al. (2015)

[9] J.A. A. Accardi, M. Anselmino, N. Armesto, E. Aschenauer, A. Bacchetta, D. Boer, W. Brooks, T. Burton, N.B. Chang, W.T. Deng et al. (2012), 1212 . 1701

[10] N. Kalantarians, J. Phys. Conf. Ser. 543, 012008 (2014)

[11] J. Maxwell, W. Detmold, R. Jaffe, R. Milner, D. Crabb, D. Day, D. Keller, O.A. Rondon, M. Jones, C. Keith et al., Jefferson Lab LOI12-14-001 (2014)

[12] A.J. Baltz, Phys. Rept. 458, 1 (2008), 0706. 3356

[13] Y.B. Yang et al., $\chi$ QCD Collaboration, in preparation (2017)

[14] W. Detmold, P.E. Shanahan, Phys. Rev. D94, 014507 (2016), 1606.04505

[15] W. Detmold, D. Pefkou, P.E. Shanahan, Phys. Rev. D95, 114515 (2017), 1703.08220

[16] F. Winter, W. Detmold, A.S. Gambhir, K. Orginos, M.J. Savage, P.E. Shanahan, M.L. Wagman, Submitted to Phys. Rev. D (2017), 1709.00395

[17] H.B. Meyer, J.W. Negele, Phys. Rev. D77, 037501 (2008), 0707 . 3225

[18] R. Horsley, R. Millo, Y. Nakamura, H. Perlt, D. Pleiter, P.E.L. Rakow, G. Schierholz, A. Schiller, F. Winter, J.M. Zanotti (UKQCD, QCDSF), Phys. Lett. B714, 312 (2012), 1205.6410

[19] C. Alexandrou, V. Drach, K. Hadjiyiannakou, K. Jansen, B. Kostrzewa, C. Wiese, PoS LATTICE2013, 289 (2014), 1311. 3174

[20] M. Deka, T. Doi, Y. Yang, B. Chakraborty, S. Dong et al., Phys.Rev. D91, 014505 (2015), 1312.4816

[21] K.F. Liu et al., PoS LATTICE2011, 164 (2011), 1203. 6388

[22] S. Dulat, T.J. Hou, J. Gao, M. Guzzi, J. Huston, P. Nadolsky, J. Pumplin, C. Schmidt, D. Stump, C.P. Yuan, Phys. Rev. D93, 033006 (2016), 1506.07443

[23] J. Ashman et al. (European Muon), Phys. Lett. B206, 364 (1988)

[24] M. Engelhardt, Phys. Rev. D95, 094505 (2017), 1701.01536

[25] X. Ji, J.H. Zhang, Y. Zhao, Phys. Rev. Lett. 111, 112002 (2013), 1304.6708

[26] D. de Florian, R. Sassot, M. Stratmann, W. Vogelsang, Phys. Rev. Lett. 113, 012001 (2014), 1404.4293

[27] M. Diehl, Phys. Rept. 388, 41 (2003), hep-ph/0307382

[28] G. Martinelli, C.T. Sachrajda, Nucl. Phys. B316, 355 (1989)

[29] G. Martinelli, C.T. Sachrajda, Phys. Lett. B196, 184 (1987)

[30] X. Ji, Phys. Rev. Lett. 110, 262002 (2013), 1305.1539

[31] Y.Q. Ma, J.W. Qiu (2014), 1404.6860 
[32] A.V. Radyushkin (2017), 1705.01488

[33] H.W. Lin, J.W. Chen, S.D. Cohen, X. Ji, Phys. Rev. D91, 054510 (2015), 1402.1462

[34] C. Alexandrou, K. Cichy, V. Drach, E. Garcia-Ramos, K. Hadjiyiannakou, K. Jansen, F. Steffens, C. Wiese, Phys. Rev. D92, 014502 (2015), 1504 . 07455

[35] B.U. Musch, P. Hagler, M. Engelhardt, J.W. Negele, A. Schafer, Phys. Rev. D85, 094510 (2012), 1111.4249

[36] M. Engelhardt, B. Musch, P. Hagler, J. Negele, A. Schafer, PoS LATTICE2012, 169 (2012)

[37] M. Engelhardt, B. Musch, T. Bhattacharya, R. Gupta, P. Hagler, J. Negele, A. Pochinsky, A. Schäfer, S. Syritsyn, B. Yoon, PoS LATTICE2014, 167 (2014)

[38] M. Engelhardt, B. Musch, P. Hägler, J. Negele, A. Schäfer, Few Body Syst. 56, 447 (2015)

[39] M. Engelhardt, B. Musch, T. Bhattacharya, R. Gupta, P. Hagler, S. Krieg, J. Negele, A. Pochinsky, S. Syritsyn, B. Yoon, PoS LATTICE2015, 117 (2016)

[40] R. Pohl, A. Antognini, F. Nez, F.D. Amaro, F. Biraben, J.M.R. Cardoso, D.S. Covita, A. Dax, S. Dhawan, L.M.P. Fernandes et al., Nature 466, 213 (2010)

[41] A. Antognini, F. Nez, K. Schuhmann, F.D. Amaro, F. Biraben, J.M.R. Cardoso, D.S. Covita, A. Dax, S. Dhawan, M. Diepold et al., Science 339, 417 (2013), http://www.sciencemag.org/content/339/6118/417.full.pdf

[42] I.T. Lorenz, H.W. Hammer, U.G. Meissner, Eur. Phys. J. A48, 151 (2012), 1205.6628

[43] R. Pohl, R. Gilman, G.A. Miller, K. Pachucki, Ann. Rev. Nucl. Part. Sci. 63, 175 (2013), 1301.0905

[44] C.E. Carlson, Prog. Part. Nucl. Phys. 82, 59 (2015), 1502.05314

[45] D.W. Higinbotham, A.A. Kabir, V. Lin, D. Meekins, B. Norum, B. Sawatzky, Phys. Rev. C93, 055207 (2016), 1510.01293

[46] R.J. Hill, EPJ Web Conf. 137, 01023 (2017), 1702.01189

[47] F. Bissey, F.G. Cao, A.R. Kitson, A.I. Signal, D.B. Leinweber, B.G. Lasscock, A.G. Williams, Phys. Rev. D76, 114512 (2007), hep-lat/0606016

[48] P. Hoodbhoy, X.D. Ji, Phys. Rev. D58, 054006 (1998), hep-ph/9801369

[49] J. Soffer, Phys. Rev. Lett. 74, 1292 (1995), hep-ph/9409254

[50] M. Gockeler, P. Hagler, R. Horsley, D. Pleiter, P.E.L. Rakow, A. Schafer, G. Schierholz, J.M. Zanotti (UKQCD, QCDSF), Phys. Lett. B627, 113 (2005), hep-lat/0507001

[51] M. Diehl, M. Göckeler, P. Hägler, R. Horsley, D. Pleiter, P.E.L. Rakow, A. Schäfer, G. Schierholz, J.M. Zanotti (UKQCD, QCDSF), Soffer bound and transverse spin densities from lattice QCD, in Transversity. Proceedings, Workshop, Como, Italy, September 7-10, 2005 (2005), pp. 173-180, hep-ph/0511032

[52] X. Artru, M. Elchikh, J.M. Richard, J. Soffer, O.V. Teryaev, Phys. Rept. 470, 1 (2009), 0802.0164

[53] R. Jaffe, private communication

[54] D. Boer, S. Cotogno, T. van Daal, P.J. Mulders, A. Signori, Y.J. Zhou, JHEP 10, 013 (2016), 1607.01654

[55] S. Cotogno, Few Body Syst. 58, 92 (2017)

[56] M. Lüscher, JHEP 08, 071 (2010), [Erratum: JHEP03,092(2014)], 1006. 4518

[57] A. Hasenfratz, F. Knechtli, Phys. Rev. D64, 034504 (2001), hep-lat/0103029

[58] C. Alexandrou, M. Constantinou, K. Hadjiyiannakou, K. Jansen, H. Panagopoulos, C. Wiese (2016), 1611.06901

[59] D.F. Geesaman, K. Saito, A.W. Thomas, Ann. Rev. Nucl. Part. Sci. 45, 337 (1995) 
[60] P.R. Norton, Rept. Prog. Phys. 66, 1253 (2003)

[61] L.B. Weinstein, E. Piasetzky, D.W. Higinbotham, J. Gomez, O. Hen, R. Shneor, Phys. Rev. Lett. 106, 052301 (2011), 1009. 5666

[62] O. Hen, G.A. Miller, E. Piasetzky, L.B. Weinstein (2016), 1611.09748

[63] J.W. Chen, W. Detmold, Phys. Lett. B625, 165 (2005), hep-ph/0412119

[64] S.R. Beane, M.J. Savage, Nucl. Phys. A761, 259 (2005), nucl-th/0412025

[65] J.W. Chen, W. Detmold, J.E. Lynn, A. Schwenk (2016), 1607.03065

[66] L. Frankfurt, M. Strikman, Physics Reports 160, 235 (1988)

[67] E. Chudakov, D. Higinbotham, C. Hyde, S. Furletov, Yu. Furletova, D. Nguyen, M. Stratmann, M. Strikman, C. Weiss, R. Yoshida, PoS DIS2016, 143 (2016), 1608.08686

[68] K. Orginos, A. Parreno, M.J. Savage, S.R. Beane, E. Chang, W. Detmold, Phys. Rev. D92, 114512 (2015), 1508.07583

[69] S.R. Beane, E. Chang, S.D. Cohen, W. Detmold, H.W. Lin, T.C. Luu, K. Orginos, A. Parreno, M.J. Savage, A. Walker-Loud (NPLQCD), Phys. Rev. D87, 034506 (2013), 1206.5219

[70] M.L. Wagman, F. Winter, E. Chang, Z. Davoudi, W. Detmold, K. Orginos, M.J. Savage, P.E. Shanahan (2017), 1706.06550

[71] S.R. Beane et al. (2017), 1705.09239

[72] K.J. Eskola, P. Paakkinen, H. Paukkunen, C.A. Salgado, Eur. Phys. J. C77, 163 (2017), 1612.05741

[73] K. Kovarik et al., Phys. Rev. D93 (2016), 1509.00792

[74] H.D. Politzer, Nucl. Phys. B172, 349 (1980)

[75] A.F. Falk, M.E. Luke, M.J. Savage, Phys. Rev. D49, 3367 (1994), hep-ph/9308288

[76] D.B. Kaplan, M.J. Savage, Phys. Lett. B365, 244 (1996), hep-ph/9509371

[77] M.P. Mattis, M. Mukherjee, Phys. Rev. Lett. 61, 1344 (1988)

[78] J. Dai, R.F. Dashen, E.E. Jenkins, A.V. Manohar, Phys. Rev. D53, 273 (1996), hep-ph/9506273

[79] R.F. Dashen, E.E. Jenkins, A.V. Manohar, Phys. Rev. D51, 3697 (1995), hep-ph/9411234

[80] R.F. Dashen, E.E. Jenkins, A.V. Manohar, Phys. Rev. D49, 4713 (1994), [Erratum: Phys. Rev.D51,2489(1995)], hep-ph/9310379

[81] R.L. Jaffe, A. Manohar, Phys. Lett. B223, 218 (1989) 